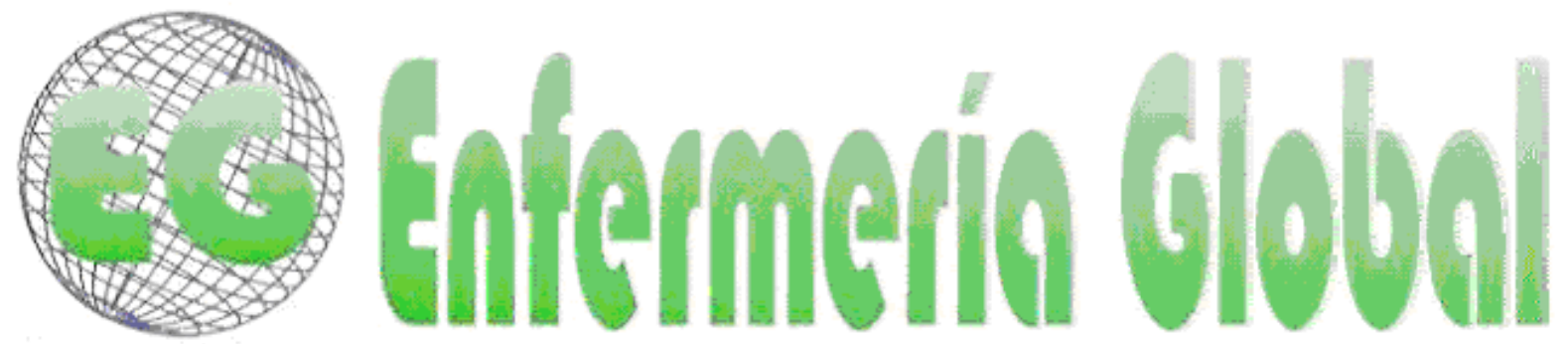

\title{
Síndrome Hellp: estudio de revisión para la atención de enfermería
}

Síndrome Hellp: estudo de revisão para o cuidado de enfermagem

\section{*De Oliveira, RS., "*De Matos, IC., **Da Silva, TBP., "De Azevedo, NM.,} ${ }^{* * *}$ Andrade, M., ${ }^{* * *}$ Do Espirito Santo, FH.

\begin{abstract}
*RN Teacher of Nursing School, Iguaçu University. Rio de Janeiro. E-mail: rsoliver@hotmail.com **RN Degree Student, Iguaçu University ***RN PhD Teacher of Nursing School, Federal Fluminense University.Rio de Janeiro. Brasil.
\end{abstract}

Palabras clave: Síndrome HELLP; Complicaciones del embarazo; Enfermería.

Palavras chave: Síndrome HELLP; Complicações da gestação; Cuidados de Enfermagem.

Keywords: Hellp syndrome; Pregnancy Complications; nursing care.

\section{RESUMEN}

Revisión narrativa de enfoque cualitativo que analiza el síndrome HELLP como una de las principales complicaciones de la hipertensión arterial en el embarazo. HELLP significa: $\mathrm{H}$ : hemolisis, $\mathrm{EL}=$ enzimas hepáticas elevadas y LP: disminución del número de plaquetas circulantes. Cuando una mujer embarazada en estado de pre-eclampsia presenta alteraciones clínicas o de laboratorio compatibles con hemolisis, se puede considerar la posibilidad de síndrome de HELLP. Cuando el cuadro materno es estable es posible seguir el embarazo con la utilización de corticoides en la inducción de la maduración del pulmón fetal, con reducción de las complicaciones maternas y neonatales, dado que en el síndrome HELLP la interrupción del embarazo es el único tratamiento capaz de disminuir el síndrome. Este estudio destaca la importancia de la propedéutica investigadora para el diagnóstico del síndrome HELLP cuando embarazadas hipertensas con pre-eclampsia son asistidas por la enfermera en las consultas de prenatal.

\section{RESUMO}

Revisão Narrativa de abordagem qualitativa que analisa a Síndrome HELLP como uma das principais complicações da hipertensão arterial na gravidez. A palavra HELLP significa: H: hemólise; EL: enzimas hepáticas elevadas e LP: diminuição do número de plaquetas circulantes. Quando uma gestante em estado de pré-eclampsia apresenta alterações laboratoriais ou clínicas compatíveis com hemólise, pode-se considerar a possibilidade da Síndrome HELLP. Quando o quadro materno apresenta-se estável é possível viabilizar a gestação a partir do uso de corticóides na indução da maturação pulmonar fetal, com redução das complicações maternas e neonatais; até porque na Síndrome HELLP o término da gestação é o único tratamento capaz de frear a Síndrome. Este estudo destaca a importância da propedêutica investigante para o diagnostico da Síndrome HELLP sempre que uma 
gestante hipertensa, portadora de pré-eclampsia é assistida pela Enfermeira nas consultas de Prénatal.

\begin{abstract}
Narrative review of a qualitative approach that examines HELLP Syndrome as a major complication of hypertension among pregnant women. It is a set of signs and symptoms that can occur in the preeclampsia. HELLP means: H: hemolysis (involves the destruction of red blood cells), EL: elevated liver enzymes and LP: decreased number of platelets (cells that help clotting) circulating. When a pregnant woman in a state of pre-eclampsia presents clinical or laboratory abnormality consistent with hemolysis one can consider the possibility of HELLP Syndrome. In the situation where a pregnant woman is stable it is possible to make the pregnancy viable with the use of steroids in the induction of fetal lung maturation, reducing the birth complications for the mother and the newborn; because in HELLP syndrome the birth is the only treatment capable of stopping it. This study highlights the importance of investigation process for the diagnosis of HELLP syndrome in a pregnant hypertensive, with preeclampsia is assisted by a nurse, to prevent the impairment of the vitality and maternal mortality in severe cases.
\end{abstract}

\title{
INTRODUCCIÓN
}

El Síndrome HELLP fue descrito inicialmente por Pritchard en 1954, y posteriormente por Louis Weinstein en 1982, quien lo denominó de HELLP. El Síndrome HELLP se considera una variante de la pre-eclampsia grave caracterizada por hemolisis, elevación de las enzimas hepáticas y plaquetopenia ${ }^{(1)(2)}$.

El presente estudio busca investigar la temática como forma de comprender e interpretar las causas de la evolución para el diagnóstico del Síndrome HELLP, así como reflexionar sobre la atención de los profesionales de salud para la detección precoz en el diagnóstico, a fin de aumentar las probabilidades de supervivencia de la madre y del bebê.

Definido como Síndrome de Hemolisis y aumento de enzimas hepáticas asociado a bajo registro de plaquetas (trombocitopenia). El síndrome HELLP se observa en mujeres grávidas con pre-eclampsia o eclampsia que manifestan alteraciones en el hígado y anormalidad en la coagulación sanguínea.

Hasta su reconocimiento como entidad específica, este Síndrome se confundía frecuentemente con cuadros de hepatitis o colecistitis, diagnosticándose erróneamente lo que provocaba retrasos en el tratamiento que culminaban en desenlace desfavorable para la gestante y el feto ${ }^{(3)}$.

A pesar de que constituye una parte en la enfermedad hipertensiva específica de la gravidez (DHEG), la condición clínica se enmarca como una etapa de agravamiento en la enfermedad separada o unida a la hipertensión arterial crónica.

\section{METODOLOGÍA}

Investigación de referencial bibliográfico con enfoque descriptivo, que permitió la comprensión e interpretación del Síndrome Hellp a partir de los estudios analizados, mostró la descripción de las características de la población susceptible al fenómeno y el establecimiento de las relaciones entre las variables del estudio ${ }^{(4)}$; realizada en el período de Junio de 2008 a Marzo de 2009, en la base de datos de BVS: LILACS (Literatura Latino-Americana y del Caribe en Ciencias de la Salud), MEDLINE (Medical Literature Analysis and Retrieval System Online), Cochrane Library, Saúde 
do Adolescente (Adolec) y Localizador de Informações em Saúde (LIS). Se utilizó el descriptor Inglés HELLP y HELLP Syndrome y el descriptor portugués: Síndrome HELLP.

El total de artículos localizados, resultantes de la búsqueda fue de 1.427 (143 en LILACS, 1.218 en MEDLINE, 46 en Cochrane, 17 en Adolec y 03 en LIS). Para establecer criterios de exclusión, se realizó un filtro en la búsqueda a partir de aquellos arículos que presentaban el descriptor en el título y disponibilidad de texto completo; encontrados 82 artículos que se identificaban con el propósito del estudio, realizada pre lectura de los resúmenes, de ellos se seleccionaron 18 artículos para análisis instrumental describiéndose en los resultados los 08 artículos que presentaron los mejores elementos y argumentos para elegir una actuación profesional segura, basada en evidencias. Utilizado como estrategia para operacionalizar el análisis y correlacionar las informaciones, un cuaderno compuesto por los ítems: tipo de estudio realizado; tamaño de la muestra; principales implicados, conclusiones de los autores, lo que permitó una mejor comprensión de los datos a partir del análisis de contenido ${ }^{(5)}$.

Una de las limitaciones de este trabajo es que los datos se analizaron a partir de las evidencias identificadas por los autores (de diferentes categorías profesionales) de los artículos seleccionados, $y$, por tanto, algunas informaciones ofrecen objetividad para la realidad de la categoría que realizó el estudio; a pesar de esta limitación, la misma no interfiere en la conclusión que busca identificar mejores evidencias para el cuidado de la enfermera a la paciente portadora de Síndrome Hellp.

\section{Incidencia, clasificación y factores de riesgo}

El Síndrome HELLP constituye patología, en el ciclo grávido puerperal, de alta morbimortalidad; una circunstancia rara, que acarrea riesgo para la vida, y que ocurre entre $0,2 \%$ a $0,6 \%$ de las gestaciones; algunas gestantes desarrollan solo una o dos de las características del Síndrome ${ }^{(3)}$.

Aunque cualquier gestante puede desarrollar el Síndrome HELLP, algunas presentan mayor riesgo incluyendo aquellas que: son blancas; tienen más de 25 años; dieron a luz anteriormente (multíparas); tienen problemas de alteración de la presión sanguínea (hipertensión arterial sistémica), presentam cuadro de pre-eclampsia o eclampsia.

La mayoría de las ocurrencias del Síndrome HELLP se produce en el tercer trimestre, aunque esta condición a veces se da en el segundo trimestre o en la semana siguiente al parto ${ }^{(8)}$. La clasificación de Tenesse $e^{(6)}$ define dos formas clínicas del Síndrome HELLP: completa y parcial. Tedesco ${ }^{(7)}$ clasifica a partir de la variación plaquetaria en grados I, II y III; los criterios utilizados para el diagnóstico y clasificación del Síndrome Hellp están descritos en la tabla siguiente. 
Tabla 1: Criterios de Forma y Clasificación del Síndrome HELLP

\begin{tabular}{l|l|l}
\hline Forma & Clase & Criterios \\
\hline Completa & I & $\begin{array}{l}\text { Plaquetas menos de } 50.000 \mathrm{~mm}^{3} \\
\text { Plaquetas entre } 50.000 \text { e } 100.000 \mathrm{~mm}^{3}\end{array}$ \\
& II & $\begin{array}{l}\text { AST } \geq 70 U \mathrm{II} / \mathrm{L} \\
\mathrm{LDH} \geq 600 \mathrm{UI} / \mathrm{I} \\
\text { Bilirrubina } \geq 1,2 \mathrm{mg} / \mathrm{dl}\end{array}$ \\
\hline Parcial & III & $\begin{array}{l}\text { Pre-eclampsia grave + ausencia de hemolisis (ELLP); } \\
\text { elevación de los marcadores hepáticos (EL). } \\
\text { Plaquetas entre } 100.000 \mathrm{~mm}^{3} \text { e } 150.000 \mathrm{~mm}^{3}\end{array}$ \\
\hline
\end{tabular}

Fuente: adaptado de Montenegro e Rezende Filho, 2008 ${ }^{(6)}$, Tedesco, $1999^{(7)}$.

\section{Cuadro clínico}

El cuadro clínico típico que ocurre en la segunda mitad de la gestación es la presencia de dolor epigástrico o en el cuadrante superior derecho ( $90 \%$ de las pacientes), particularmente asociada a náuseas y vómitos ( $50 \%$ de las pacientes), hemorragia gastrointestinal y edema ${ }^{(1)}$. Es importante recordar que la hipertensión y la proteinúria pueden estar ausentes o ser levemente anormales. Algunas pacientes pueden presentar señales y síntomas, ninguno de ellos característico de pre-eclampsia grave; por eso, se recomienda análisis de laboratorio para todas las gestantes con alguno de estos síntomas ${ }^{(8)}$.

Aunque la causa del Síndrome HELLP todavía no está completamente definida, la misma puede acarrear insuficiencia cardíaca y pulmonar, hemorragia interna, accidente vascular cerebral y otras complicaciones graves para la madre. Asimismo puede ocasionar el desprendimiento prematuro de la placenta, de la pared uterina, lo que puede acabar en muerte fetal; otras complicaciones para el feto son el crecimiento uterino limitado y SARA (Síndrome de angustia respiratoria) ${ }^{(8)}$.

\section{Conducta}

EI Síndrome HELLP es un estado grave que requiere una evaluación materno fetal completa; 3 conductas deben ser consideradas: La primera es la interrupción inmediata de la gestación, auxiliada por cuidados intensivos; está indicada en las siguientes situaciones: Presencia de eclampsia asociada; Evidencia de sufrimiento fetal agudo; Evidencia de coagulopatía materna; HELLP Clase I; y en otras complicaciones asociadas a la pre-eclampsia como hipertensión refractaria, insuficiencia renal, inminencia de eclampsia y en gestación de más de 34 semanas $^{(8)(9)}$. El tiempo correcto para la interrupción de un concepto viable depende de una serie de factores de la madre, del feto y del tipo y condiciones de la asistencia obstétrica, clínica y neonatal ofrecida. La interrupción de la gestación en el Síndrome HELLP no es una indicación absoluta de césarea aunque en la mayoría de los casos (64 a $87 \%$ ) esta sea la via elegida para la interrupción de la gestación. En presencia de hematoma hepático se impone la realización del parto por vía alta debido al riesgo de ruptura y hemorragia intraperitoneal ${ }^{(8)}$. En las gestaciones con prematuridad extrema (edad gestacional por debajo de 24 semanas) la inducción del parto y la vía escogida será la que el cuadro materno permita ${ }^{(8)}$. Cualquier conducta que no sea la intervencióno inmediata debe ser orientada según la viabilidad fetal y edad gestacional $^{(1)}$. 
La segunda posibilidad de actuación seríaa una conducta semi-intervencionista que tenga como objetivo tomar medidas que puedan tener un impacto en la supervivencia y disminución de la morbidad neonatal visto que $80 \%$ de los casos ocurren en gestaciones pre-término; esta conducta se basa en el uso de corticoides administrados a la madre con el objetivo de acelerar la maturidade pulmonar fetal, reducir la incidencia de hemorragia intraventricular neonatal y de mejorar la adaptación hemodinámica del recién nacido pre-término; la administración profiláctica de corticoides está indicada entre 24 y 34 semanas de gestación; esta conducta se basa en los estudios relacionados con la administración de corticoides a altas dosis y mejora del cuadro del paciente ${ }^{(1)(2)}$.

La tercera conducta considerada conservadora en el Síndrome HELLP es controvertida y restringida a un grupo muy selectivo de pacientes con gestacióno pretérmino, mantenidas bajo condiciones de monitorización materno-fetal ideales y en centros terciarios. Datos preliminares de un estudio multicéntrico europeo mostraron una incidencia significativamente mayor de complicaciones maternas graves (edema pulmonar, insuficiencia renal y alteraciones hemorrágicas) en pacientes sometidas a conducta expectante en comparación con el tratamiento semi-conservador y /o imediato $^{(1)}$.

\section{RESULTADOS}

Para una mejor comprensión de los resultados presentados, según los aspectos diferenciales de los estudios investigados, estos se ordenaron según el tipo de estudio, el año de realización, así como las principales conclusiones de los autores.

Se seleccionaron tres relatos de casos clínicos; dos estudios de caso; dos revisiones de literatura y un ensayo clínico. En 2005, Oliveira ${ }^{(10)}$ registró los criterios de laboratorio para el diagnóstico y clasificación del Síndrome HELLP. Refiere que los profesionales que realizan acompañamiento pre-natal deben estar atentos a la sintomatología para que no haya demora en el diagnóstico y prejuicio para la madre y o para el feto; porque el tratamiento es específico para cada situación y exige estrecha relación interdisciplinar entre los profesionales involucrados. La Unidad de salud, de selección del ingreso, debe estar preparada para: realizar intervenciones quirúrgicas, Terapia Intensiva madre-bebé o enviar a centros especializados. En 2004 Castro $^{(11)}$ mostró que el diagnóstico precoz permite el tratamiento específico en tiempo hábil, reduciendo la morbi-mortalidad y alterando el curso natural de la enfermedad; y en 1998 Peraçoli $^{(12)}$ mostró que la incidencia de Síndrome HELLP fue del $0,3 \%$ y su recurrencia sucedió en el $16,7 \%$ de los casos; que cuando se diagnostica la hipertensión arterial, se debe proceder a monitorización periódica de la variación sistólica/diastólica y de análisis de laboratorio; el profesional debe estar atento a las alteraciones que caracterizan el Síndrome HELLP y, así, intervenir antes que haya manifestación completa, tornando sombrío el pronóstico materno-fetal.

Fueron dos estudios de serie de casos; en 2007 Angonesi ${ }^{(13)}$ investigó a 13 pacientes, $(8,44 \%)$ de 154 prontuarios analizados y que fueron diagnosticados con Síndrome HELLP. Una de ellas $(7,7 \%)$ tuvo gestación gemelar y hubo un óbito fetal; cuatro pacientes $(30,76 \%)$ fueron hemotransfundidas con plaquetas; concluyó que no existe consenso, en relación a los valores de laboratorio para el diagnóstico del Síndrome HELLP parcial. En el estudio no hubo óbito materno, solo un óbito fetal $(7,7 \%)$. Refiere que la mortalidad materna varía de 3,4\% a $24,2 \%$ y la mortalidad perinatal, de $8 \%$ a $60 \%$. En 2006, Peres ${ }^{(14)}$ investigó 12 casos de un total de 6.000 partos $(0,2 \%)$; 
donde la insuficiencia renal aguda se diagnosticó en 8 pacientes (66,7\%). Los recién nacidos presentaban peso medio de $1.500 \mathrm{~g}$. El Síndrome de membrana hialina sucedió en el $25 \%$ de los casos, la tasa de mortalidad fue de $33,4 \%$. Concluyó que el Síndrome HELLP es la más frecuente causa de insuficiencia renal aguda gestacional y presenta una alta morbi-mortalidad materna y perinatal, donde el diagnóstico precoz y el tratamiento especializado pueden reducir la morbi-mortalidad.

Se seleccionaron dos estudios de revisión de Literatura: en 2007 Orcy $^{(15)}$ identifica factores de riesgo de Síndrome Hellp como: mujeres negras (con aumento de la edad materna), índice de masa corporal, resistencia a insulina, historia familiar, factores genéticos, tabaquismo. Concluye que identificar riesgos y marcadores biológicos precozmente es fundamental para la prevención o reducción de los riesgos de mortalidad materna y fetal. En 2006 Santos $^{(16)}$ sugiere que el equipo de enfermería debe estar atento a todos los acontecimentos y dudas de la gestante, disminuyendo su sufrimiento a través de la orientación y ayuda, por ello Enfermería precisa de conocimientos y sensibilidad para identificar, entender y acompañar el proceso fisiológico y emocional que interviene en la gestación de alto riesgo ${ }^{(17)}$.

En $2007 \mathrm{Katz}^{(18)}$ realizó un ensayo clínico cuya media de edad de las pacientes era de 26,7 años. El diagnóstico (Síndrome HELLP) se realizó en el pre-parto en 45,2\% de los casos; las complicaciones encontradas fueron manifestaciones hemorrágicas: $34,3 \%$, oligúria en $46,7 \%$ e insuficiencia renal aguda en $20 \%$; la hemotransfusión fue necesaria en 33,3\%; que 6,7\% presentaron edema agudo de pulmón; y 3,8\% (4 casos) acabaron en muerte. Mostró que el tiempo medio entre el diagnóstico y el alta u óbito era de 10,3 días. Caracterizó el Síndrome HELLP como una dolencia grave, de elevada morbi-mortalidad; que los cuidados intensivos y procedimientos especiales aumentan el período de ingreso y costos hospitalarios.

\section{Comentarios}

Los estudios en su mayoría son recientes, con menos de 05 años de su publicación, excepto un estudio de caso que fue relacionado por la riqueza de los datos presentados y que contribuyó de forma importante a las reflexiones de este estudio.

En todos los estudios el diagnóstico se fundamentó en criterios de laboratorio bien establecidos, mostrando las evidencias de anemia hemolítica, aumento de las enzimas hepáticas y bajo número de plaquetas; como describen los conceptos clásicos de la literatura. A pesar de que los análisis de laboratorio son confiables y ampliamente aceptados como indicadores de la instalación del Síndrome Hellp, la sintomatología enmascara las evidencias y no siempre los profesionales reconocen prontamente su presencia; enfermedades como gastritis, apendicitis, cálculos renales, hepatitis, presentan informes clínicos y de laboratorio semejantes y tiende a ser la primera sospecha diagnóstica ${ }^{(2)}$.

En el tratamiento de pacientes con hipertensión se debe considerar la edad gestacional y el riesgo de pre-eclampsia y su gravedad para la elección de la conducta más adecuada ${ }^{(9)(19)}$. La detección precoz del Síndrome HELLP aumenta las posibilidades de supervivencia de la madre y del bebé; este es el motivo por el cual la enfermera debe percibir inmediatamente cualquier síntoma de anormalidad durante las consultas de enfermería de prenatal. La demora en el diagnóstico favorece la aparición de complicaciones serias como insuficiencia renal aguda, edema agudo de pulmón o ruptura hepática, que pueden llevar a la muerte. Es necesario el 
acompañamiento y vigilancia permanente de la paciente grávida con variación de la presión significativa; porque la enfermedad hipertensiva de la gestación es una de las principales causas de mortalidad materna.

De ocurrencia rara, este Síndrome constituye cuadro patológico que se presenta como factor contribuyente para los índices de mortalidad materna y perinatal; la mortalidad materna (aceptable hasta 20/100.000, por la Organización Mundial de la Salud), en Brasil es considerada alta, pues las tasas varían de 75 a 110/100.000 nacidos vivos ${ }^{(19)}$.

Considerando que el Síndrome HELLP es un episodio grave, de inicio imprevisto, con mal pronóstico, y que tiene posibilidad de repetirse en gestaciones futuras, es importante que las gestantes reciban orientación en cuanto a la gravedad de la enfermedad de la probabilidad de recurrencia de la misma, además de iniciar el acompañamiento de pre-natal precoz, como factor de impacto positivo sobre la calidad de vida de las gestantes ${ }^{(20)}$.

\section{CONCLUSIÓN}

El Síndrome HELLP abarca una extensa condición clínica pudiendo mimetizar o ser mimetizadas por una serie de complicaciones clínicas asociadas o agravadas por la gravidez. La pre-eclampsia es factor de riesgo para enfermedad cardiovascular futura incluso aunque la primera gravidez fue normal. En estas condiciones, cualquier preeclampsia en la gravidez es indicación para el cambio en el estilo de vida (dieta, control de peso, ejercicios). En la práctica todavía no se ha conseguido prevenir o al menos reducir la incidencia o alterar la evolución de la dolencia. Cuando el Síndrome HELLP ocurre antes de la $30^{\underline{a}}$ semana de gestación, la conducta obstétrica queda condicionada por la presencia de las complicaciones y la evaluación clínica y laboratorial. Casos considerados de alto riesgo para la madre justifican la interrupción; los casos bajo control permiten que la gravidez evolucione hasta la madurez fetal. En la evaluación del mínimo riesgo materno y de la máxima supervivencia fetal, el equipo debe considerar las condiciones hospitalarias, laboratoriales y de nido.

Así, reconociendo que la mortalidad materna no puede ser solo un dato estadístico ${ }^{(21)}$ y que constituye un desafío que debe involucrar a toda la Sociedad, el Ministerio de Salud ha incentivado la formación de Comités para la Reducción de la Mortalidad Materna ${ }^{(19)}$, como estrategia para variar este cuadro, además de adoptar una serie de medidas para mejorar la calidad de la asistencia obstétrica a través de la participación en compromisos internacionales.

De este modo, la Enfermera, durante la Consulta de Enfermería de acompañamiento de prenatal, debe estar atenta a la aparición de manifestaciones clínicas ligadas a la hipertensión ${ }^{(22)}$, hemorragias, sangrados y principalmente la presencia de signos y síntomas de pre-eclampsia, para así intervenir en este processo para disminuir las complicaciones y mortalidad asociada.

\section{REFERENCIAS BIBLIOGRÁFICAS}

1. Cavalcante J. Gravidez de alto risco. $2^{\circ}$ ed. São Paulo: Difusão 2003. 
2. Haram K, Svendsen E, Abildgaard U. The HELLP syndrome: Clinical issues and management. A Review. BNC Pregnancy Childbirth 2009 9(8): 1-15. available at http://www.biomedcentral.com/1471-2393/9/8 acesso em 18/03/2009.

3. Chaves Netto H. Obstetrícia Básica, 2a Ed. São Paulo: Atheneu, 2004.

4. Minayo MCS, Deslandes SF, (Orgs.) Caminhos do Pensamento: Epistemologia e Método. Rio de Janeiro: Fiocruz; 2002. 380 p.

5. Bardin L. Análise de Conteúdo. Trad. Luis Antero Reto e Augusto Pinheiro, Lisboa: Edições 70. 2008. 281 p.

6. Montenegro CAB, Rezende Filho J. Obstetrícia Fundamental, 11ª Ed. Rio de Janeiro: Guanabara Koogan, 2008.

7. Tedesco JJA. A grávida suas indagações e as dúvidas do obstetra. São Paulo: Atheneu 1999.

8. Peracoli JC, Parpinelli MA. Síndromes hipertensivas da gestação: identificação de casos graves. Rev. Bras. Ginecol. Obstet., out. 2005, vol.27, no.10, p.627-634.

9. Amorim MMR, Katz L, Ávila MB, Araújo DE, Valença M, Albuquerque CJM, et al. Perfil das admissões em uma unidade de terapia intensiva obstétrica de uma maternidade brasileira. Rev. Bras. Saúde Matern. Infant., Recife, 6 (Supl 1): S55S62, maio, 2006. disponivel em http://www.scielo.br/pdf/rbsmi/v6s1/30505.pdf acesso em 20/12/2009.

10. Oliveira AC, Vasconcelos VO, Fernandes JHA. Ruptura da cápsula hepática em Hellp síndrome. Revista Medica Ana Costa. 2005; 10(4) out-dez05, disponível em http://www.revistamedicaanacosta.com.br/10(4)/artigo 6.htm acesso em 23/10/2008.

11. Castro RF, Maia FFR, Ferreira AR, Purisch S, Calsolari MR, Menezes PAFC, Victória IMN. HELLP Síndrome Associada à Síndrome de Cushing - Relato de Dois Casos. Arq Bras Endocrinol Metab vol 48 n 3 Junho 2004. disponível em http://www.scielo.br/pdf/abem/v48n3/a15v48n3.pdf, acesso em 23/10/2008.

12. Peraçoli JC, Rudge MVC, Calderon IMP, Maestá I, Sgarbosa F. Síndrome Hellp recorrente: relato de dois casos. Rev. Bras. Ginecol. Obstet. 1998; 20 (3): 165-167. disponível em http://www.scielo.br/pdf/rbgo/v20n3/a08v20n3.pdf acesso em 25/10/2008.

13. Angonesi, Janaína; Polato, Angelita. Doença hipertensiva específica da gestação (DHEG), incidência à evolução para a Síndrome de HELLP. Rev. Bras. Anal.Clin; 2007; 39(4):243-245. disponível em http://www.sbac.org.br/pt/pdfs/rbac/rbac 39 04/rbac 3904 01.pdf, acesso em 23/10/2008.

14. Peres LAB, Katarinhuk BSLACS. Avaliação epidemiológica da ocorrência de insuficiência renal aguda na Síndrome de Hellp em um Hospital Geral no Oeste do Paraná. J Bras Nefrol. 2006; 28(3): 139-143. disponível em http://www.jbn.org.br/28-3/04-Peres-28(3).pdf acesso em 22/10/2008.

15. Orcy RB, Pedrini R, Piccinini P, Schroeder S, Costa SHM, Ramos JGL, Capp E, Corleta HVE. Diagnóstico, fatores de risco e patogênese da pré-eclâmpsia. Revista HCPA, Vol. 27, No 3 (2007). disponível em http://www.seer.ufrgs.br/index.php/hcpa/article/view/1981/1579, acesso em 22/10/2008

16. Santos E, Silva FE, Nakamura EK. A importância do esclarecimento da Enfermagem para a gestante de alto risco e baixa renda. Revista de Enferm Centro Universitário Campos de Andrade. 2008; Disponível em http://www.uniandrade.edu.br/links/menu3/publicacoes/revista enfermagem/artigo 08.pdf acesso em 23/10/2008.

17. Costa IG. As percepções da gravidez de risco para a gestante e as implicações familiares. Rev Gaucha Enferm. (internet) 2002; 23 (1) p. 4391/2337. disponível 
em

http://www.seer.ufrgs.br/index.php/RevistaGauchadeEnfermagem/article/view/4391 /2337 acesso em 12/03/2010.

18. Katz L, Amorim MMR, Miranda GV, Silva JLP. Perfil clínico, laboratorial e complicações de pacientes com síndrome HELLP admitidas em uma unidade de terapia intensiva obstétrica. Rev Bras Ginecol Obstet. 2008; 30(2):80-6. disponível em http://www.scielo.br/pdf/rbgo/v30n2/06.pdf, acesso em 22/11/2008.

19. Brasil, Ministério da Saúde/Secretaria de Políticas de Saúde/Área Técnica de Saúde da Mulher. Manual dos Comitês de Mortalidade Materna, $2^{a}$ Edição. Brasília; 2002.

20. Godoy SR, Bergamasco RB, Gualda DMR, Tsunechiro MA. Severe obstetric morbidity - near miss. Meaning for surviving women: oral history. Online Braz $\mathrm{J}$ Nurs (periódico na Internet) 2008; 7(2): (aproximadamente 8 p.) disponível em http://www.uff.br/objnursing/index.php/nursing/article/view/ j.16764285.2008.1460/372.

21.Pelloso S, Tavares MSG. A problemática familiar e a morte materna. Ciênc Cuid Saúde, 2006; v.5, supl., p.19-25. Disponível em: http://periodicos.uem.br/ojs/index.php/CiencCuidSaude/article/view/5148/3334 Acesso em: 23 abr. 2010.

21. Ximenes Neto FR, Melo JR. High-blood pressure control in primary health care: na analysis of Nurses Pratices. Enfermería Global [periódico na internet] 2005; may (6): 506-552. disponível em http://revistas.um.es/eglobal/article/viewFile/506/552 